
Tom Houben, Ronit Shiri-Sverdlov*

\title{
Insulin resistance is positively associated with plasma cathepsin D activity in NAFLD patients
}

https://doi.org/10.1515/bmc-2021-0011

received May 23, 2021; accepted July 13, 2021.

\begin{abstract}
Previous studies associated plasma cathepsin D (CTSD) activity with hepatic insulin resistance in overweight and obese humans. Insulin resistance is a major feature of non-alcoholic fatty liver disease (NAFLD) and is one of the multiple hits determining the progression towards non-alcoholic steatohepatitis (NASH). In line, we have previously demonstrated that plasma CTSD levels are increased in NASH patients. However, it is not known whether insulin resistance associates with plasma CTSD activity in NAFLD. To increase our understanding regarding the mechanisms by which insulin resistance mediates NAFLD, fifty-five liver biopsy or MRI-proven NAFLD patients $\left(\mathrm{BMI}>25 \mathrm{~kg} / \mathrm{m}^{2}\right)$ were included to investigate thelink between plasma CTSDactivity to insulin resistance in NAFLD. We concluded that HOMA-IR and plasma insulin levels are independently associated with plasma CTSD activity in NAFLD patients (standardized coefficient $\beta$ : 0.412, 95\% Cl: 0.142 0.679, $\mathrm{p}=0.004$ and standardized coefficient $\beta: 0.495,95 \% \mathrm{Cl}: 0.236 \sim 0.758$, $\mathrm{p}=0.000$, respectively). Together with previous studies, these data suggest that insulin resistance may link to NAFLD via elevation of CTSD activity in plasma. As such,
\end{abstract}

\footnotetext{
*Corresponding author: Ronit Shiri-Sverdlov, Department of Molecular Genetics, NUTRIM School of Nutrition and Translational Research in Metabolism, Maastricht University Medical Center+, Universiteitssingel 50, 6229ER Maastricht, the Netherlands, E-mail: r.sverdlov@maastrichtuniversity.nl Lingling Ding, Yvonne Oligschlaeger, Tom Houben, Department of Molecular Genetics, NUTRIM School of Nutrition and Translational Research in Metabolism, Maastricht University Medical Center+, Universiteitssingel 50, 6229ER Maastricht, the Netherlands Toon. J. I. De Munck, Ger. H. Koek, Department of Internal Medicine, NUTRIM School of Nutrition and Translational Research in Metabolism, Maastricht University Medical Center+, Universiteitssingel 50, 6229ER Maastricht, the Netherlands Jef Verbeek, Department of Gastroenterology \& Hepatology, University Hospitals KU Leuven, Leuven, Belgium

Ger. H. Koek, Department of visceral and transplantation surgery, Klinikum, RWTH, Aachen, Germany
}

these data pave the way for testing CTSD inhibitors as a pharmacological treatment of NAFLD.

Keywords: Insulin; HOMA-IR; lysosomal enzyme; plasma CTSD activity; NAFLD; NASH.

\section{Introduction}

Non-alcoholic fatty liver disease (NAFLD) is a disease spectrum, which ranges from simple steatosis to non-alcoholic steatohepatitis (NASH) (steatosis and inflammation with or without fibrosis), liver cirrhosis and hepatocellular carcinoma [1]. While the pathogenesis of NAFLD has been extensively studied, the mechanisms underlying NAFLD and its progression are far from being clarified [2], resulting in the lack of well-defined effective ways to prevent NAFLD development and progression.

One of the key factors that contributes to the transition from NAFLD progression to NASH is insulin resistance [3]. Relevantly, previous research in our group showed that plasma activity of thelysosomal enzyme cathepsin D(CTSD) is inversely associated with hepatic insulin sensitivity in overweight and obese individuals [4]. Moreover, we have previously demonstrated that plasma CTSD levels are increased in NASH patients [5, 6], thereby linking CTSD to a progressed NAFLD state. The positive correlation between plasma CTSD and hepatic inflammation on one hand, and the inverse correlation between hepatic insulin sensitivity and plasma CTSD activity in overweight and obese humans on the other hand suggest that insulin resistance is positively associated with plasma CTSD activity in NAFLD patients. However, it is not yet known whether insulin resistance associates with plasma CTSD activity in NAFLD. Investigating the link between plasma CTSD activity to insulin resistance in NAFLD patients will increase our understanding regarding the mechanisms by which insulin resistance mediates NAFLD.

In the current paper, plasma CTSD activity and insulin resistance-related parameters were determined in 
fifty-five NAFLD patients. Afterwards, the multiple linear regressions were performed to analyze the associations between parameters of insulin resistance and plasma CTSD activity with adjustment for age, sex, BMI and waist circumference.

\section{Methods}

\section{Subjects characteristics}

Fifty-five patients were enrolled in our study including 29 men and 26 women, with an age ranging from 20 to 65 years and BMI $\geq 25 \mathrm{~kg} / \mathrm{m}^{2}$. All subjects were proven NAFLD by liver biopsy (based on the criteria of NAS (NAFLD activity score)) or chemical shift MRI (magnetic resonance imaging MRI). Metabolic syndrome (MetS) was determined according to the definition of International Diabetes Federation (IDF) [7]. Subjects were selected based on exclusion criteria including consuming excessive ethanol (male $\geq 14$ and female $\geq 7$ standard beverages in one week), suffering from secondary hepatic fat accumulation (causes as previously described [8]), a history of bariatric surgery, liver cirrhosis and/or hepatocellular carcinoma as well as extrahepatic malignancy(s) within the past 5 years. Besides, subjects who are pregnant and breastfeeding, use insulin and are about to undergo or recovering from a surgical /medical procedure were also ruled out from our study. All participants were collected from Maastricht University Medical Centre (MUMC+) and CO-EUR (a second line eating disorder clinic) during the period September 2015-October 2018.

Informed consent: Informed consent has been obtained from all individuals included in this study.

Ethical approval: The research related to human use has been complied with all the relevant national regulations, institutional policies and in accordance the tenets of the Helsinki Declaration (revised in 2008), and approved by the Medical-Ethical Committee of Maastricht University with ClinicalTrials.gov Identifier: NCT02422238.

\section{Biochemical analyses}

Blood samples were drawn after overnight fasting at Maastricht University Medical Center (MUMC+). All blood samples were centrifuged to yield plasma (1000 x g; 10 $\min ; 4^{\circ} \mathrm{C}$ ) and then stored at $-80^{\circ} \mathrm{C}$ freezer until analyses. Plasma samples were further analyzed at the clinical chemistry department of Maastricht UMC+ hospital to determine aspartate aminotransferase (AST), alanine aminotransferase (ALT), $y$-glutamyl transpeptidase (GGT), bilirubin, alkaline Phosphatase, total cholesterol, triglyceride, low density lipoprotein cholesterol (LDLcholesterol) and high density lipoprotein cholesterol (HDL-cholesterol) as previously described [8]. Besides, fasting plasma glucose, hemoglobin $\mathrm{A} 1 \mathrm{c}$ ( $\mathrm{HbA1C}$ ) and plasma insulin levels were evaluated using routine laboratory methods. Insulin resistance was calculated by (fasting plasma insulin $(\mathrm{mU} / \mathrm{L}) \times$ fasting plasma glucose $(\mathrm{mmol} / \mathrm{L})) / 22.5$ according to the homeostasis model of assessment-insulin resistance (HOMA-IR) [9]. Plasma CTSD activity was evaluated using a CTSD activity assay kit by following manufacturer's protocol (MBL International, Woburn, USA) as previously described [4]. To each assay, $5 \mu \mathrm{L} 10 \mathrm{x}$ diluted plasma sample was added in black 96-well plate and $52 \mu \mathrm{L}$ mastermix ( $50 \mu \mathrm{L}$ CTSD reaction buffer and $2 \mu \mathrm{L}$ CTSD substrate) was added. After mixing, the 96-well plate was incubated at $37^{\circ} \mathrm{C}$ for $2 \mathrm{~h}$ (protection from light) and then the plate was measured via using a fluorescence plate reader with 328-nm excitation wavelength and 460-nm emission wavelength. The activity of plasma CTSD was expressed by the relative fluorescence units.

\section{Magnetic Resonance Imaging}

The hepatic fat fraction (steatosis) was determined by using chemical shift gradient echo MRI. Four circular region of interests (ROIs) of $5 \mathrm{~cm}^{2}$ from three MRI sections were drawn in the liver, where artefact, vascular and biliary structures were avoided. Then, the ROI was copied from the in-phase (IP) image to the opposed-phase (OP) image and the average ROI signal intensity (SI) for the IP and OP image were calculated. At the end, the mean signal intensity loss of all 12 ROIs was determined by using the formula: (SI IP- SI OP)/ 2^SI IP*100\% [10].

\section{Data statistics}

Statistical analyses were performed by using SPSS 25.0 (IBM, Armonk, NY, USA) for Microsoft Windows. All data were expressed as mean $\pm \mathrm{SD}$. A bivariate correlation was used to analyze the simple correlations between plasma CTSD activity and other parameters. Then multiple linear regression analyses were conducted for analyzing the association between plasma CTSD activity and insulin/ HOMA-IR, where plasma CTSD activity was added as dependent variable and either HOMA-IR or insulin as independent variables generating model 1 (simple 
regression), model 2 (model $1+$ correcting for age), model 3 (model $2+$ correcting for sex), model 4 (model 3 + correcting for BMI) and model 5 (model $4+$ correcting for waist circumference). $p$-value $<0.05$ was statistically significant. Interactions between co-variates (age, sex, BMI and waist circumference) in the multiple regression analyses were also tested but no significant interactions were found (data not shown).

\section{Results}

\section{General clinical characteristics of the study participants}

The baseline characteristics of the population are listed in Table 1. Fifty-five NAFLD patients were included in our study, including 29 males and 26 females with an average age of 51.4 years old and mean BMI of $32.3 \mathrm{Kg} /$ $\mathrm{m}^{2}$. The hepatic fat fraction (\%) ranged from 5.2 to $45.5 \%$. Furthermore, plasma CTSD activity ranged from 93.9 RFU/ $\mu \mathrm{L}$ to $324.3 \mathrm{RFU} / \mu \mathrm{L}$, with a mean of $179.6 \pm 58.9 \mathrm{RFU} / \mu \mathrm{L}$.

\section{HOMA-IR and plasma insulin levels are positively associated with plasma CTSD activity}

To investigate whether insulin resistance-related parameters correlate with plasma CTSD activity in NAFLD patients, multiple linear regression analyses were performed. As shown in Table 2, HOMA-IR was positively associated with plasma CTSD activity (Model 1: standardized $\beta=0.413,95 \% \mathrm{Cl}$ : 0.156 0.667, $\mathrm{p}=0.002$ ), even after adjustment for age (Model 2: standardized $\beta=0.417$, 95\% Cl: 0.162 0.669, $\mathrm{p}=0.002$ ), sex (Model 3: standardized $\beta=0.394,95 \% \mathrm{Cl}: 0.129 \sim 0.656, \mathrm{p}=0.004$ ), BMI (Model 4: standardized $\beta=0.410,95 \% \mathrm{Cl}: 0.143 \sim 0.674, \mathrm{p}=0.003)$ and waist circumference (Model 5: standardized $\beta=0.412$, 95\% $\mathrm{Cl}$ : 0.142 0.679, $\mathrm{p}=0.004)$. Furthermore, we found that plasma insulin levels were also positively associated with plasma CTSD activity (Model 1: standardized $\beta=0.472$, 95\% Cl: 0.225 0.723, $\mathrm{p}=0.000$ ), independent of age (Model 2: standardized $\beta=0.487,95 \% \mathrm{Cl}: 0.243 \sim 0.735, \mathrm{p}=0.000$ ), sex (Model 3: standardized $\beta=0.468,95 \% \mathrm{Cl}: 0.214 \sim 0.726$, $\mathrm{p}=0.001$ ), BMI (Model 4: standardized $\beta=0.493$, 95\% $\mathrm{Cl}$ : 0.237 0.753, $\mathrm{p}=0.000$ ) and waist circumference (Model 5: standardized $\beta=0.495,95 \% \mathrm{Cl}$ : 0.236 0.758, $\mathrm{p}=0.000$ ). Our data therefore demonstrate that insulin resistance is
Table 1: General clinical characteristics of the study participants.

\begin{tabular}{lll}
\hline & Mean \pm SD & Ranges \\
\hline Age, yrs & $51.4 \pm 11.7$ & $20 \sim 65$ \\
Sex (M/F) & $29 / 26$ & - \\
BMI, kg/m & $32.3 \pm 4.9$ & $24.6 \sim 46.3$ \\
Waist circumference, cm & $106.8 \pm 11.8$ & $82.0 \sim 146.9$ \\
Hip circumference, cm & $112.4 \pm 11.9$ & $96.0 \sim 154.3$ \\
SBP (mmHg) & $135.0 \pm 15.1$ & $104 \sim 177$ \\
DBP (mmHg) & $81.1 \pm 9.6$ & $64 \sim 113$ \\
Heart rate (bpm) & $67.6 \pm 11.4$ & $49 \sim 100$ \\
MetS (Yes/No) & $31 / 24$ & - \\
MetS (points) & $3.0 \pm 1.6$ & $0 \sim 5$ \\
HbA1c (\%) & $5.8 \pm 0.8$ & $4.6 \sim 8.8$ \\
Fasting glucose, mmol/L & $6.2 \pm 1.4$ & $4.6 \sim 11.7$ \\
Insulin, pmol/L & $99.3 \pm 65.8$ & $17.7 \sim 364.0$ \\
HOMA-IR & $4.2 \pm 3.6$ & $0.6 \sim 23.8$ \\
Total Cholesterol (mmol/L) & $5.2 \pm 1.0$ & $3.3 \sim 7.7$ \\
Triglycerides (mmol/L) & $2.1 \pm 2.2$ & $0.7 \sim 15.6$ \\
HDL Cholesterol (mmol/L) & $1.3 \pm 0.4$ & $0.5 \sim 2.2$ \\
LDL Cholesterol (mmol/L) & $3.2 \pm 0.9$ & $0.9 \sim 5.4$ \\
GGT (U/L) & $54.6 \pm 46.3$ & $12 \sim 256$ \\
ALT (U/L) & $51.2 \pm 43.8$ & $7 \sim 272$ \\
AST (U/L) & $33.9 \pm 19.6$ & $11 \sim 118$ \\
Bilirubin ( $\mu$ mol/L) & $9.3 \pm 4.8$ & $3.7 \sim 28.7$ \\
Alkaline phosphatase (IU/L) & $95.0 \pm 30.0$ & $52 \sim 186$ \\
Hepatic steatosis (\%) & $19.1 \pm 10.3$ & $5.2 \sim 45.5$ \\
CTSD activity, RFU/ $\mu$ L & $179.6 \pm 58.9$ & $93.9 \sim 324.3$ \\
\hline & & \\
\hline
\end{tabular}

Data are expressed as mean \pm SD. BMI, body mass index; WHR, waist/hip ratio; SBP, systolic blood pressure; DBP, diastolic blood pressure.

independently associated with plasma CTSD activity in the context of NAFLD.

\section{Discussion}

We here show that plasma insulin levels and HOMA-IR are independently associated with plasma CTSD activity in NAFLD patients. Together with previous studies, our finding suggests that insulin resistance may be linked to NAFLD via elevation of plasma CTSD activity. 
Table 2: HOMA-IR and plasma insulin levels are positively associated with plasma CTSD activity independent of age, sex, BMI and waist circumference.

\begin{tabular}{|c|c|c|c|c|c|c|c|}
\hline \multicolumn{8}{|c|}{ Dependent variable: Plasma CTSD activity } \\
\hline \multicolumn{4}{|l|}{ HOMA-IR } & \multicolumn{4}{|c|}{ Plasma insulin levels (Insulin) } \\
\hline Models & $\begin{array}{l}\text { Adjusted } \\
\text { R square }\end{array}$ & $\begin{array}{l}\text { Standardized } \\
\text { coefficient } \beta \\
(95 \% \mathrm{Cl}) \\
\end{array}$ & $p$ value & Models & $\begin{array}{l}\text { Adjusted } \\
\text { R square }\end{array}$ & $\begin{array}{l}\text { Standardized } \\
\text { coefficient } \beta \\
(95 \% \mathrm{Cl}) \\
\end{array}$ & $p$ value \\
\hline Model 1 & 0.154 & & & Model 1 & 0.208 & & \\
\hline HOMA-IR & & $\begin{array}{l}0.413 \\
(0.156 \sim 0.667)\end{array}$ & 0.002 & Insulin & & $\begin{array}{l}0.472 \\
(0.225 \sim 0.773)\end{array}$ & 0.000 \\
\hline Model 2 & 0.167 & & & Model 2 & 0.231 & & \\
\hline HOMA-IR & & $\begin{array}{l}0.417 \\
(0.162 \sim 0.669)\end{array}$ & 0.002 & Insulin & & $\begin{array}{l}0.487 \\
(0.243 \sim 0.735)\end{array}$ & 0.000 \\
\hline Age & & $\begin{array}{l}0.169 \\
(-0.084 \sim 0.418)\end{array}$ & 0.188 & Age & & $\begin{array}{l}0.196 \\
(-0.049 \sim 0.435)\end{array}$ & 0.115 \\
\hline Model 3 & 0.158 & & & Model 3 & 0.221 & & \\
\hline HOMA-IR & & $\begin{array}{l}0.394 \\
(0.129 \sim 0.656)\end{array}$ & 0.004 & Insulin & & $\begin{array}{l}0.468 \\
(0.214 \sim 0.726)\end{array}$ & 0.001 \\
\hline Age & & $\begin{array}{l}0.193 \\
(-0.071 \sim 0.452)\end{array}$ & 0.149 & Age & & $\begin{array}{l}0.213 \\
(-0.041 \sim 0.462)\end{array}$ & 0.099 \\
\hline Sex & & $\begin{array}{l}-0.095 \\
(-0.369 \sim 0.178)\end{array}$ & 0.485 & Sex & & $\begin{array}{l}-0.074 \\
(-0.338 \sim 0.139)\end{array}$ & 0.572 \\
\hline Model 4 & 0.159 & & & Model 4 & 0.230 & & \\
\hline HOMA-IR & & $\begin{array}{l}0.410 \\
(0.143 \sim 0.674)\end{array}$ & 0.003 & Insulin & & $\begin{array}{l}0.493 \\
(0.237 \sim 0.753)\end{array}$ & 0.000 \\
\hline Age & & $\begin{array}{l}0.181 \\
(-0.084 \sim 0.441)\end{array}$ & 0.177 & Age & & $\begin{array}{l}0.200 \\
(-0.053 \sim 0.468)\end{array}$ & 0.120 \\
\hline Sex & & $\begin{array}{l}-0.064 \\
(-0.345 \sim 0.216)\end{array}$ & 0.646 & Sex & & $\begin{array}{l}-0.036 \\
(-0.305 \sim 0.234)\end{array}$ & 0.791 \\
\hline BMI & & $\begin{array}{l}-0.132 \\
(-0.388 \sim 0.129)\end{array}$ & 0.319 & BMI & & $\begin{array}{l}-0.158 \\
(-0.404 \sim 0.094)\end{array}$ & 0.276 \\
\hline Model 5 & 0.141 & & & Model 5 & 0.214 & & \\
\hline HOMA-IR & & $\begin{array}{l}0.412 \\
(0.142 \sim 0.679)\end{array}$ & 0.004 & Insulin & & $\begin{array}{l}0.495 \\
(0.236 \sim 0.758)\end{array}$ & 0.000 \\
\hline Age & & $\begin{array}{l}0.190 \\
(-0.093 \sim 0.468)\end{array}$ & 0.184 & Age & & $\begin{array}{l}0.211 \\
(-0.060 \sim 0.476)\end{array}$ & 0.125 \\
\hline Sex & & $\begin{array}{l}-0.086 \\
(-0.448 \sim 0.275)\end{array}$ & 0.632 & Sex & & $\begin{array}{l}-0.062 \\
(-0.408 \sim 0.284)\end{array}$ & 0.720 \\
\hline BMI & & $\begin{array}{l}-0.086 \\
(-0.608 \sim 0.438)\end{array}$ & 0.745 & BMI & & $\begin{array}{l}-0.104 \\
(-0.602 \sim 0.398)\end{array}$ & 0.684 \\
\hline $\begin{array}{l}\text { Waist } \\
\text { circumference }\end{array}$ & & $\begin{array}{l}-0.053 \\
(-0.608 \sim 0.499)\end{array}$ & 0.844 & $\begin{array}{l}\text { Waist } \\
\text { circumference }\end{array}$ & & $\begin{array}{l}-0.063 \\
(-0.594 \sim 0.464)\end{array}$ & 0.805 \\
\hline
\end{tabular}

Data were analyzed by multiple linear regression models: Model 1, simple regression; Model 2, model $1+$ adjustment for age; Model 3 , model 2 + adjustment for sex; Model 4, model 3 + adjustment for BMI; Model 5, model $4+$ adjustment for waist circumference. $p<0.05$ is statistically significant. 
While insulin resistance is mostly associated with type 2 diabetes, its pathophysiology is known as an important mediator towards more severe states of NAFLD progression such as non-alcoholic steatohepatitis (NASH) [11]. Additionally, NAFLD patients seem to show increased plasma CTSD activity when compared to a previous cohort of healthy individuals [12]. Our current observation that plasma CTSD activity associates with insulin resistance in this NAFLD cohort therefore suggests that plasma CTSD might be linked to NAFLD by regulating insulin resistance. This view is in line with previous studies by us and others that demonstrate that plasma CTSD mediates insulin signaling $[4,13,14]$, hepatic inflammation $[6,13$, $15]$ and lipotoxicity $[13,15]$, all aspects that are involved with insulin sensitivity $[16,17]$. However, we like to clarify that our findings only provide initial evidences for a link between insulin resistance, plasma CTSD activity and NAFLD (and not NAFLD progression per se). As such, further in-depth investigation to validate our current observations is essential. Of note, it is important to mention that we cannot identify NAFLD progression in the current cohort. Therefore, future studies should be performed in liver biopsy-proven NAFLD patients to assess whether our claimed association also impact NAFLD progression. Nevertheless, these data support the approach to test CTSD inhibitors as a pharmacological treatment of NAFLD. A limitation of the current study is that the cohort is relatively small. Future studies that include larger patient cohorts are therefore warranted to validate our findings.

Acknowledgements: This study gets funding supports from the Dutch Organization for Scientific Research (NWO) (Vidi grant no. 016.126.327), ASPASIA (grant no. 015.008.043), TKI-LSH (grant no. 40-41200-98-9306) and VCK (grant no. Swu16.0057-VT). Lingling Ding is supported by the Chinese Scholarship Council with file number 201709110146.

Author contributions: RS and TH were responsible for concept and design of study and final critical review of manuscript. LD and TJID were responsible for drafting manuscript, data collection, statistical analysis, and critical review of manuscript. YO, JV and GHK were responsible for data collection and interpretation of data. All the authors have approved the final manuscript.

Conflicts of interests: The authors have declared that no competing interests exist.
Data Availability Statement: The datasets generated during and analysed during the current study are available from the corresponding author on reasonable request.

\section{References}

1. Hardy T, Oakley F, Anstee QM, Day CP. Nonalcoholic Fatty Liver Disease: Pathogenesis and Disease Spectrum. Annu Rev Pathol. 2016 May;11(1):451-96.

2. Tarantino G, Citro V, Capone D. Nonalcoholic Fatty Liver Disease: A Challenge from Mechanisms to Therapy. J Clin Med. 2019 Dec;9(1):E15.

3. Buzzetti E, Pinzani M, Tsochatzis EA. The multiple-hit pathogenesis of non-alcoholic fatty liver disease (NAFLD). Metabolism. 2016 Aug;65(8):1038-48.

4. Ding LL, et al. Plasma cathepsin D activity is negatively associated with hepatic insulin sensitivity in overweight and obese humans. Diabetologia. 2019.

5. Walenbergh SM, Houben T, Rensen SS, Bieghs V, Hendrikx T, van Gorp PJ, et al. Plasma cathepsin D correlates with histological classifications of fatty liver disease in adults and responds to intervention. Sci Rep. 2016 Dec;6(1):38278.

6. Walenbergh SM, Houben T, Hendrikx T, Jeurissen ML, van Gorp PJ, Vreugdenhil AC, et al. Plasma cathepsin D levels: a novel tool to predict pediatric hepatic inflammation. Am J Gastroenterol. 2015 Mar;110(3):462-70.

7. Alberti KG, Zimmet P, Shaw J. Metabolic syndrome-a new worldwide definition. A Consensus Statement from the International Diabetes Federation. Diabet Med. 2006 May;23(5):469-80.

8. Ding L, De Munck TJ, Oligschlaeger Y, Dos Reis IM, Verbeek J, Koek GH, et al. Myosteatosis in NAFLD patients correlates with plasma Cathepsin D. Biomol Concepts. 2021 May;12(1):27-35.

9. Matthews DR, Hosker JP, Rudenski AS, Naylor BA, Treacher DF, Turner RC. Homeostasis model assessment: insulin resistance and beta-cell function from fasting plasma glucose and insulin concentrations in man. Diabetologia. 1985 Jul;28(7):412-9.

10. Cassidy FH, Yokoo T, Aganovic L, Hanna RF, Bydder M, Middleton MS, et al. Fatty liver disease: MR imaging techniques for the detection and quantification of liver steatosis. Radiographics. 2009 Jan-Feb;29(1):231-60.

11. Utzschneider KM, Kahn SE. Review: the role of insulin resistance in nonalcoholic fatty liver disease. J Clin Endocrinol Metab. 2006 Dec;91(12):4753-61.

12. Ding L, Houben T, Oligschlaeger $\mathrm{Y}$, Bitorina AV, Verwer BJ, Tushuizen ME, et al. Plasma Cathepsin D Activity Rather Than Levels Correlates With Metabolic Parameters of Type 2 Diabetes in Male Individuals. Front Endocrinol (Lausanne). 2020 Sep;11:575070.

13. Khurana P, Yadati T, Goyal S, Dolas A, Houben T, Oligschlaeger Y, et al. Inhibiting Extracellular Cathepsin D Reduces Hepatic Steatosis in SpraguelDawley Rats t. Biomolecules. 2019 May;9(5):E171.

14. Liu L, Chen B, Zhang X, Tan L, Wang DW. Increased Cathepsin D Correlates with Clinical Parameters in Newly Diagnosed Type 2 Diabetes. Dis Markers. 2017;2017:5286408. 
15. Houben T, Oligschlaeger Y, Hendrikx T, Bitorina AV, Walenbergh SM, van Gorp PJ, et al. Cathepsin D regulates lipid metabolism in murine steatohepatitis. Sci Rep. 2017 Jun;7(1):3494.

16. Savage DB, Petersen KF, Shulman GI. Disordered lipid metabolism and the pathogenesis of insulin resistance. Physiol Rev. 2007 Apr;87(2):507-20.

17. de Luca C, Olefsky JM. Inflammation and insulin resistance. FEBS Lett. 2008 Jan;582(1):97-105. 